\title{
Rest Frame Optical Spectra of Lyman Break Galaxies: Other Lensing Arcs around MS1512-cB58
}

\author{
Matthew A. Malkan*, Harry I. Teplitz ${ }^{\dagger}$ and Ian S. McLean* \\ *UCLA Astronomy, LA CA 90095-1562 \\ ${ }^{\dagger}$ SIRTF Science Center, Pasadena CA 91125
}

\begin{abstract}
We have obtained near-infrared spectra of two images of the galaxy at $z=2.72$ which is gravitationally lensed by the foreground cluster MS1512+36. The brighter arc, cB58, is an image of only the nucleus and the southern half of the background galaxy, while the fainter image, A2, encompasses the entire background galaxy. Thus the gravitational lensing provides spatial resolution on a smaller scale than is routinely available by other methods.

Our observations indicate no evidence for any systematic rotational velocity gradient across the face of this galaxy. The nucleus and outer regions of the galaxy do not differ in their gas reddening or excitation level, based on the identical $\mathrm{H} \alpha / 5007$ ratios. cB58 (which is more dominated by the nucleus) has relatively stronger continuum emission, perhaps because of a higher ratio of old to young stars, compared to the outer parts of the galaxy.

A second emission line source, denoted as K1, at a slightly lower redshift was serendipitously detected in the slit. It appears to be the gravitationally lensed image of another background galaxy in the same group as cB58.
\end{abstract}

\section{A SECOND IMAGE OF THE BRIGHTEST KNOWN LBG}

Most of the detailed spectral information on $z>2$ galaxies has come from observation of the rest-frame ultraviolet redshifted into the optical passband. The Lyman Break Galaxies (LBGs; [1]) are strongly starbursting galaxies, and in principle may be the tracers of the global star formation history of the universe, if the effects of dust extinction on the UV continuum can be quantified. However, the average attenuation from dust in these galaxies is uncertain to almost an order of magnitude ([2], [3]): ([4]) suggest a factor of $>6$, while [5] argue no correction is needed.

The need for measurements in the optical rest frame is clear, but even with the advent of IR spectrographs on large telescopes, only about two dozen $z>2$ starbursts have been spectroscopically observed in the rest frame optical (e.g. [2], [6], [7]).

The $\mathrm{z}=2.72$ galaxy MS1512-cB58 ([8]) is the apparently brightest Lyman Break Galaxy known, due to lensing magnification (of 50X) by a foreground galaxy cluster at $\mathrm{z}=0.37$. Lensing reconstruction ([9]) shows that $\mathrm{cB} 58$ is a magnification of only half of the source galaxy, and parts of the nucleus are not included. The brightest counter arc to cB58, "A2", is a magnification of a the entire source. Thus spectroscopy of the two arcs provides measurement of different spatial regions in the source galaxy. This kind of high spatial resolution is not routinely available to intrinsically faint, unlensed, LBGs even with AO. 


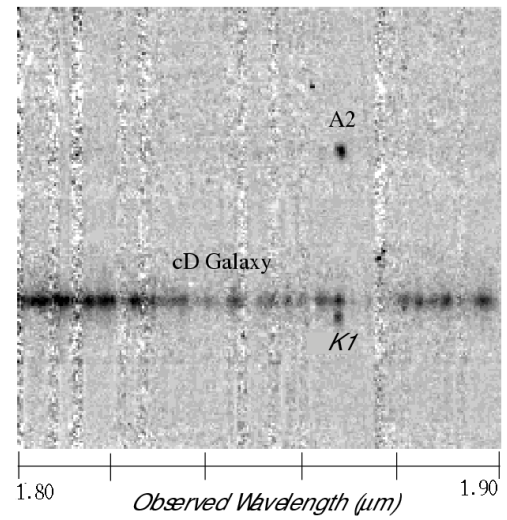

FIGURE 1. The two dimensional, rectified, atmospheric absorption corrected spectrum of A2 and other objects in the slit. The strong line in the A2 spectrum is redshifted [OIII]5007. The object labeled K1 was not previously known. Note that the apparent bump at a similar wavelength in the continuum of the cD galaxy is not an emission line, but merely a local maximum in the atmospheric transmission which, unlike the new redshifted 5007 line, goes away when divided by an A star spectrum.

Keck-II Infrared Spectroscopy. On 4 April 2002, we used NIRSPEC ([10]) to obtain a spectrum of $\mathrm{A} 2$, in the $\mathrm{H}$ and $\mathrm{K}$ bands $(1.5-2.5 \mu \mathrm{m})$, with $\mathrm{R} \sim 1500$ (FWHM $\sim 4$ pixels $=$ 16 Angstroms). In 20 and 30 minutes of observation in $\mathrm{H}$ and $\mathrm{K}$ respectively, we clearly detect the OIII doublet (see figures 1 and 2) and $\mathrm{H} \alpha$. However, $\mathrm{H} \beta$ falls partially on a night sky line.

\section{COMPARISON OF THE CB-58 ARC AND ITS COUNTER-ARC A2}

Redshift: The redshift calculated from the emission lines in cB58 is $2.7290 \pm 0.0007$, in contrast to the $z_{a b s}=2.7233 \pm 0014$ reported from the rest-frame UV interstellar absorption lines ([8]). This systematic shift is typical LBGs ([2],[5]). The velocity of A2 is almost identical (within the noise of the A2 spectrum, $\Delta V \leq 100 \mathrm{~km} / \mathrm{sec}$.) indicating a narrow range of radial velocities across the entire object.

Line Widths: Emission lines allow us to measure the velocity dispersion. Using the lensing reconstruction, we obtain a virial mass $M_{v i r}=1.0 \times 10^{10} \mathrm{M}_{\odot}$ for $\mathrm{cB} 58$, in good agreement with the masses of LBGs at $z \sim 3$ ([5]). None of the emission lines in A2 are resolved in velocity (due to the use of a wider slit), consistent with the small FWHM measured for $\operatorname{cB} 58(\Delta v \sim 175 \mathrm{~km} / \mathrm{sec})$

Extinction and Chemical Abundance: In cB58 we measure $\mathrm{H} \alpha: \mathrm{H} \beta=3.23$ implying $\mathrm{E}(\mathrm{B}-\mathrm{V}) \simeq 0.27$ using the [11] reddening law (and very little change using the LMC law). Its derived one-third solar metallicity is consistent with the $Z / Z_{\odot} \sim 0.25$ found by [12] and 0.4 by [13]. Since the [OIII] $5007 / \mathrm{H} \alpha$ ratio in both cb58 and A2 is the same (0.85 for cB58 and $0.89+/-0.06$ for A2), there is no evidence for any large-scale spatial variation of extinction or metallicity across this galaxy. 

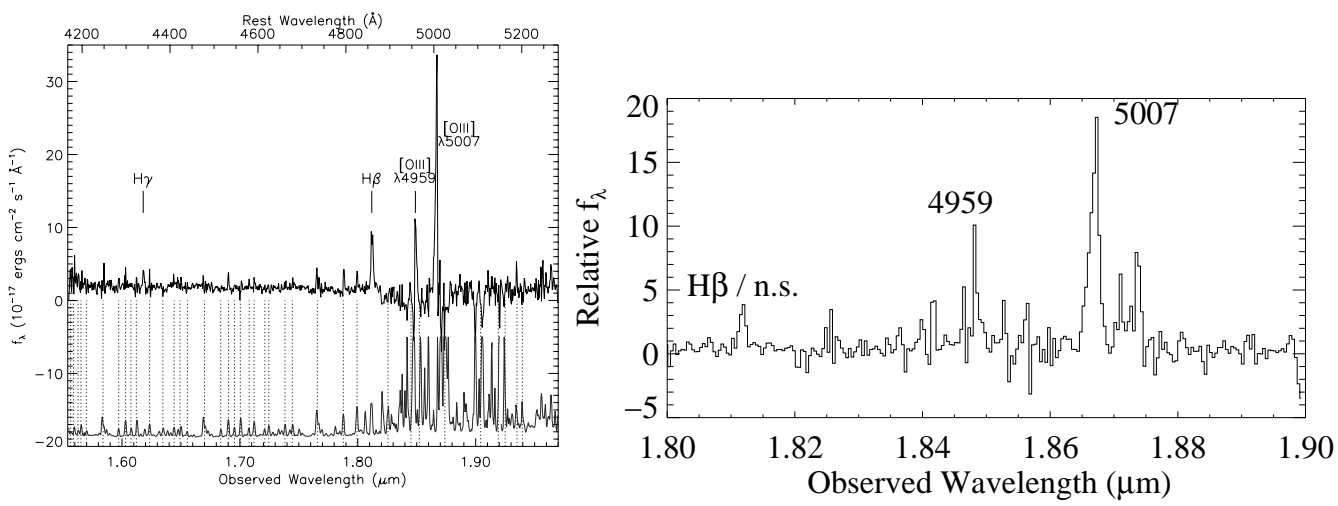

FIGURE 2. left: The H-band spectrum of the main arc, cB58. The $1 \sigma$ error bars are plotted underneath, showing the sharp increase longward of $1.8 \mu \mathrm{m}$, where the atmospheric transmission cuts off. right: The extracted H-band spectrum of the counter-arc image A2.

Star Formation Rate: The most dramatic difference between our spectra of the cB58 arc and the A2 image is that the latter, which includes more of nucleus of the lensed galaxy, shows twice as large equivalent widths of $\mathrm{H} \alpha$ and OIII. The local star formation is proceeding at different rates in different parts of this object.

\section{SERENDIPITOUS DISCOVERY OF A NEW ARCLET AT Z=2.69}

In addition to A2, we serendipitously detected a new emission line source in the field. Figure 3 shows the location of the NIRSPEC slit we used to measure the A2 candidate, superposed on the F555W HST image of the field. On the other side of the cD galaxy, which we used for the alignment and setup of the slit, we found another high-z lineemitting galaxy; this object, "K1", was independently confirmed by the detection of both $\mathrm{H} \alpha$ and OIII5007 in nodded pairs of spectra. The two dimensional spectrum shows the clearly detected 5007 line in the counter arc (A2) and K1. [OIII]4959 is detected, but $\mathrm{H} \beta$ falls on an $\mathrm{OH}$ line and is not convincingly detected above the noise in this short (20 minutes) integration.

\section{Comparison of $\mathrm{K} 1$ to $\mathrm{cB58} / \mathrm{A} 2$}

$\mathrm{K} 1$ is blue-shifted from cB58 and A2 by $\sim 150 \mathrm{~km} / \mathrm{sec}$. The $\mathrm{H} \alpha /[\mathrm{OIII}]$ ratio is much lower in K1, $0.25 \pm 0.05$, which could be explained by some combination of lower reddening and/or lower metal abundance. K1 is likely to be a lensed image of a different 


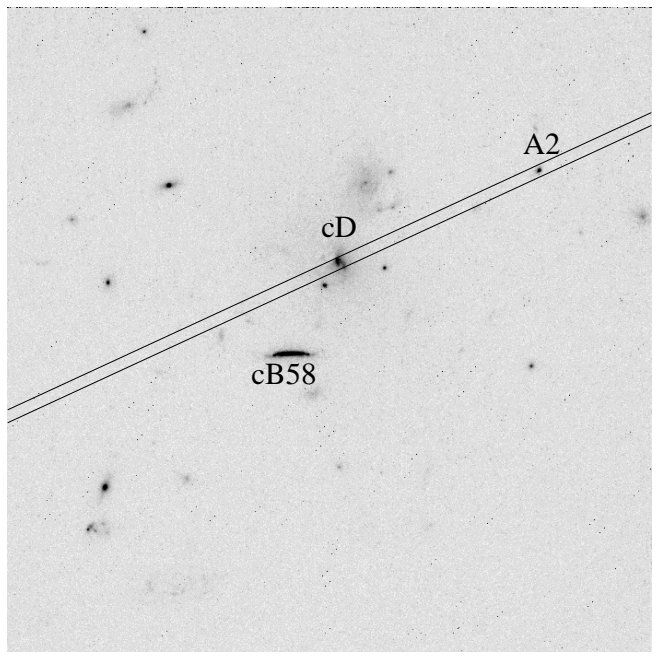

FIGURE 3. The NIRSPEC slit, superposed on the F555W WPFC2 image of the field. The image is trimmed to $32 " x 32 "$. North is down.

galaxy in the same group, rather than another counter image of cB58.

\section{ACKNOWLEDGMENTS}

We thank Stella Seitz for useful discussions.

\section{REFERENCES}

1. Steidel, C.C., Giavalisco, M., Pettini, M., Dickinson, M., \& Adelberger, K.L., 1996, ApJ Letters 462, L17

2. Pettini, M., Kellogg, M., Steidel, C.C., Dickinson, M., Adelberger, K.L., \& Giavalisco, M., 1998, ApJ 508, 539; $\mathrm{P} 98$

3. Trager, S.C., Faber, S.M., Dressler, A., \& Oemler, A., 1997, ApJ 485, 92

4. Meurer, G.R., Heckman, T.M., \& Calzetti, D. 1999, ApJ, 521, 64

5. Pettini, M. et al. 2001, ApJ, 554, 981

6. Teplitz, H., et al. 2000, ApJL, 533, 65

7. Pettini, M. et al. 2002, ApJ, 569, 742

8. Yee, H.K.C., Ellingson, E., Bechtold, R.G., Carlberg,R.G., Cuillandre, J.-C., 1996, AJ 111, 1783

9. Seitz, S., Saglia, R., Bender, R., Hopp, U., Belloni, P., Ziegler, B., 1998, MNRAS 298, 945

10. McLean, I. S., et al. 1998, SPIE, vol. 3354, 566

11. Calzetti, D.A., Kinney, A.L., \& Storchi-Bergmann, T., 1994, ApJ, 429, 482

12. Pettini, M. et al. 2000, ApJ, 528, 96

13. Frayer, D.T., et al. 1997, AJ, 113, 562 